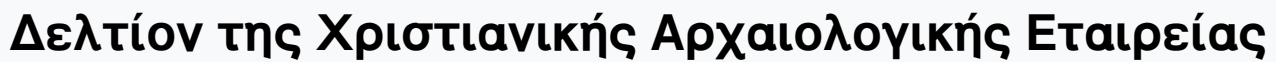

Tó 30 (2009)

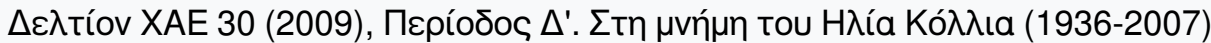

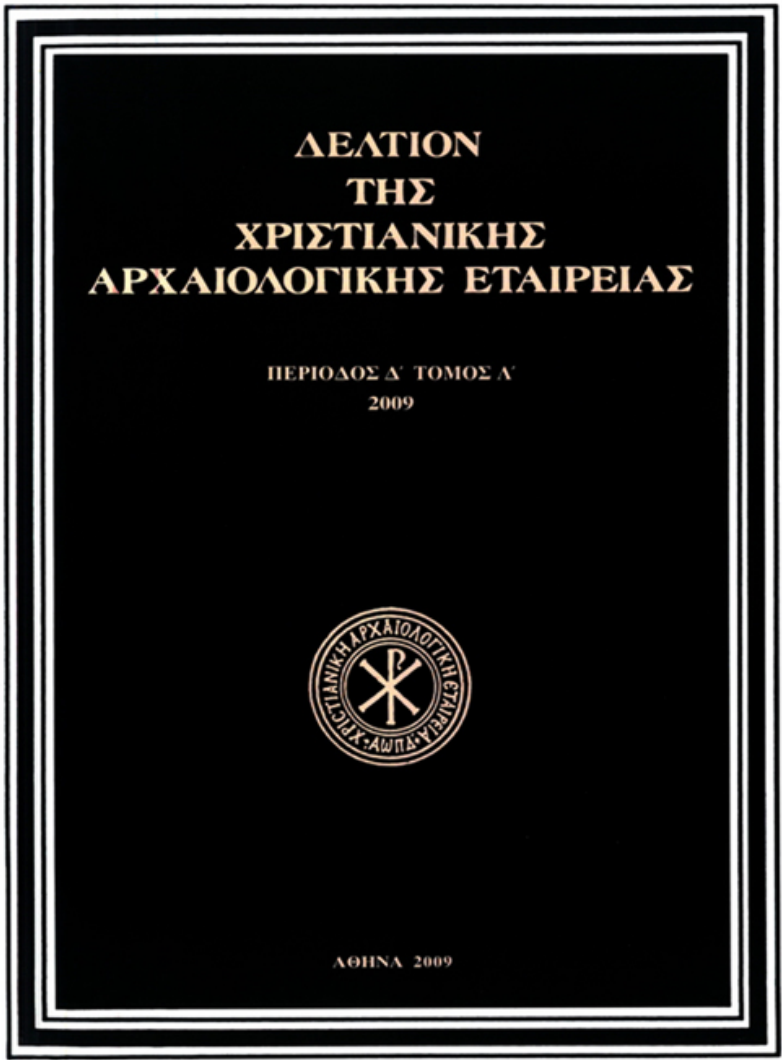

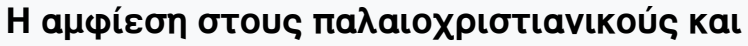

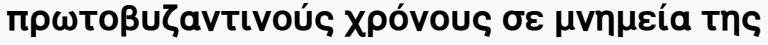

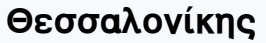

\section{EUTÉрпп MAPKH}

doi: $10.12681 /$ dchae.659

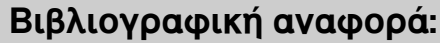

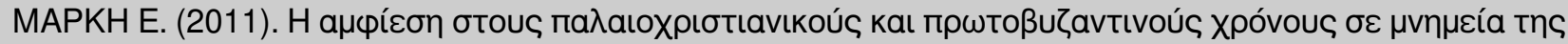

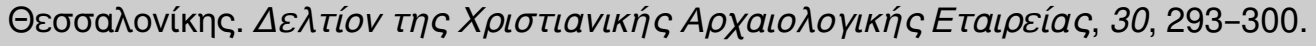

https://doi.org/10.12681/dchae.659 


\section{$\triangle \mathrm{E} \Lambda \mathrm{TION}$ TH $\Sigma$ XPI $\Sigma$ TIANIKH $\Sigma$ APXAIO $\Lambda$ OГIKH $\Sigma$ ETAIPEIA $\Sigma$}

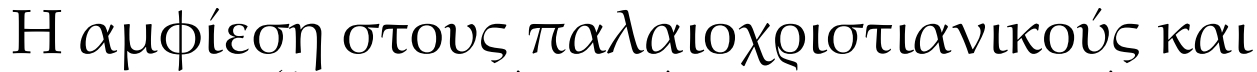

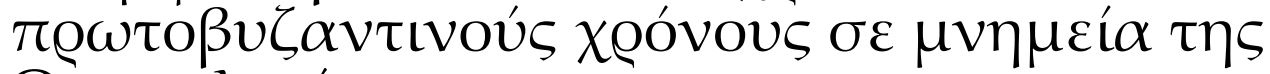
$\Theta \varepsilon \sigma \sigma \alpha \lambda$ ovíkฺs

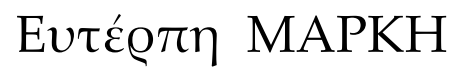

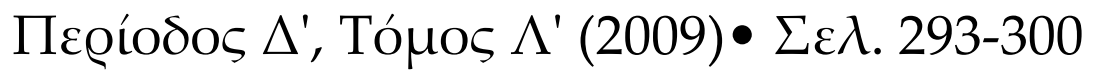
A@HNA 2009 


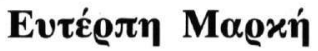

\section{H AMФIE $\Sigma$ H $\Sigma$ TOY $\Sigma$ ПA АAIOXPI $\Sigma$ TIANIKOY $\Sigma$ KAI ПPQTOBYZANTINOY $\Sigma$ XPONOY $\Sigma$ $\Sigma$ E MNHMEIA TH $\Sigma$ $\Theta E \Sigma \Sigma$ A $\Lambda$ ONIKH $\Sigma$}

$\mathrm{T}_{\mathrm{a}}$

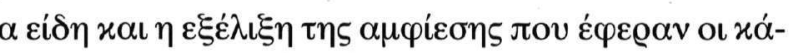

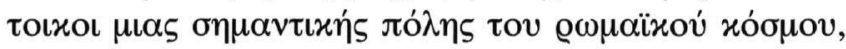

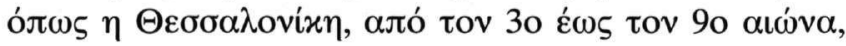

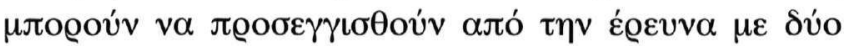

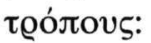

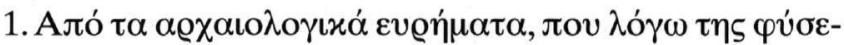

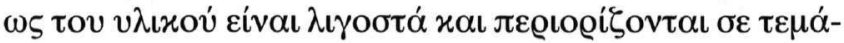

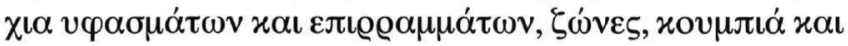

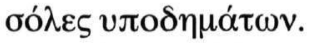

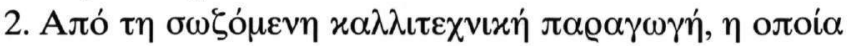

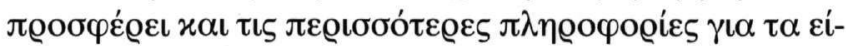

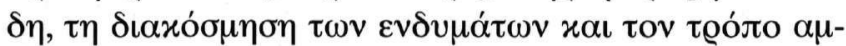

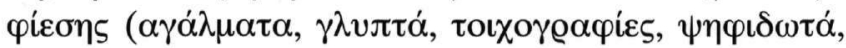

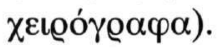

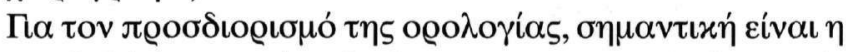

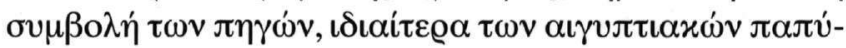

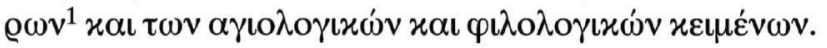

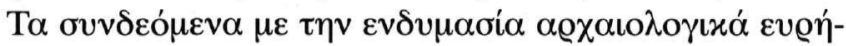

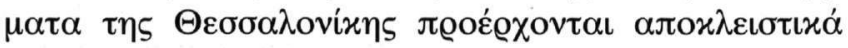

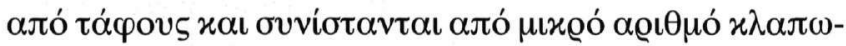

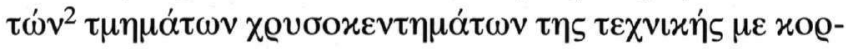

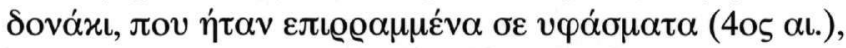

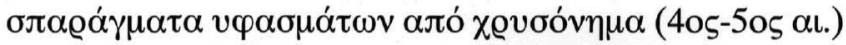

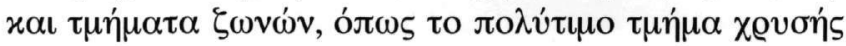

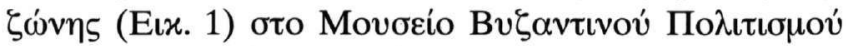

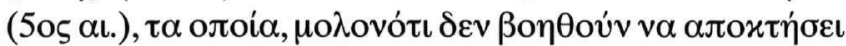

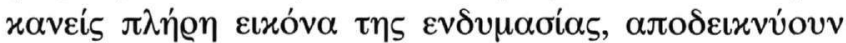

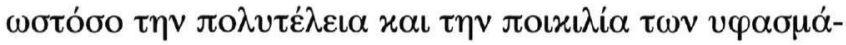

\footnotetext{
${ }^{1}$ Select Papyri, Loeb Classical Library ( $\mu \tau \varphi \varrho$. A. S. Hunt $\varkappa \alpha \iota$ C. C. Edgar).

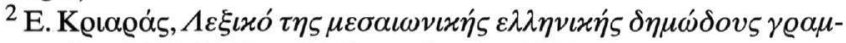

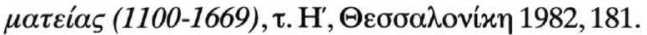

${ }^{3}$ Select Papyri, ó. $\pi ., \alpha \varrho \iota \theta .182$.

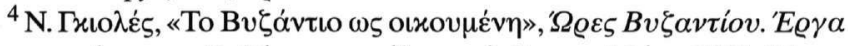

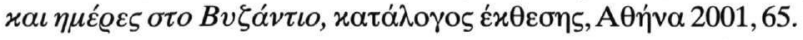

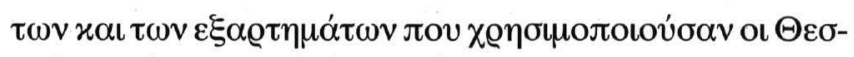

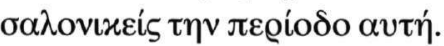

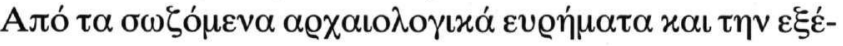

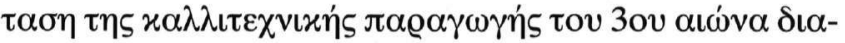

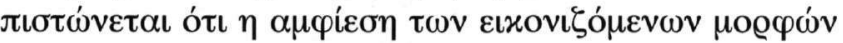

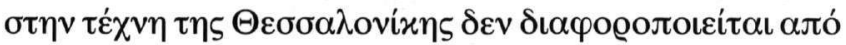

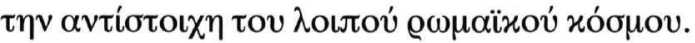

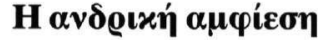

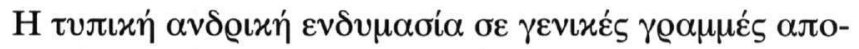
$\tau \varepsilon \lambda \varepsilon i \tau \alpha \iota$ aлó:

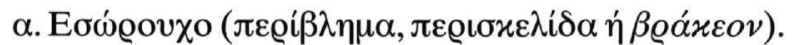

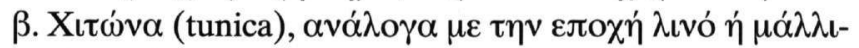

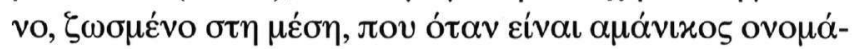

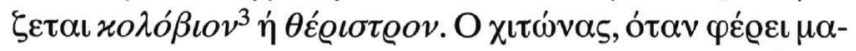

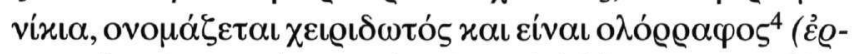

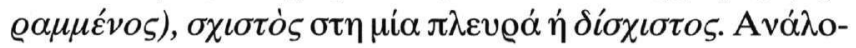

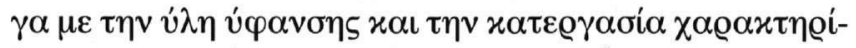

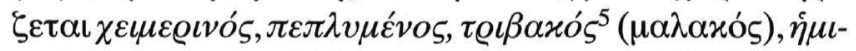

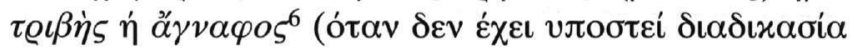
$\lambda \varepsilon v \dot{x a v \sigma \eta \zeta) . ~}$

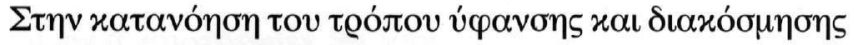

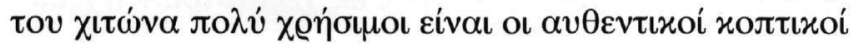

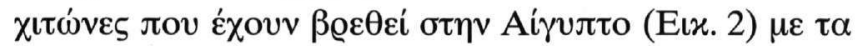

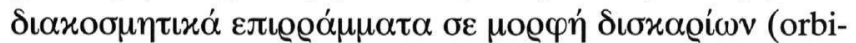

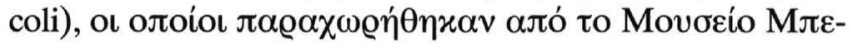

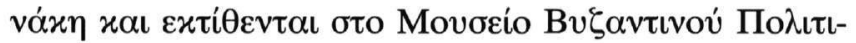

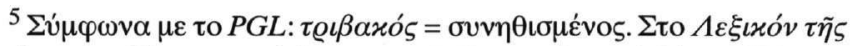

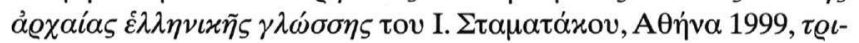

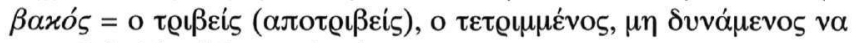

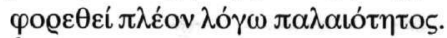

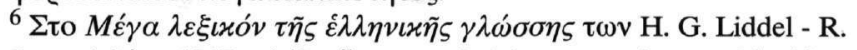

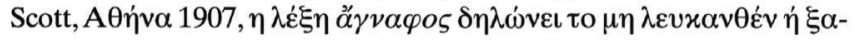

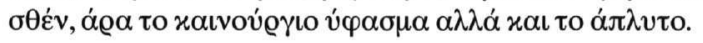




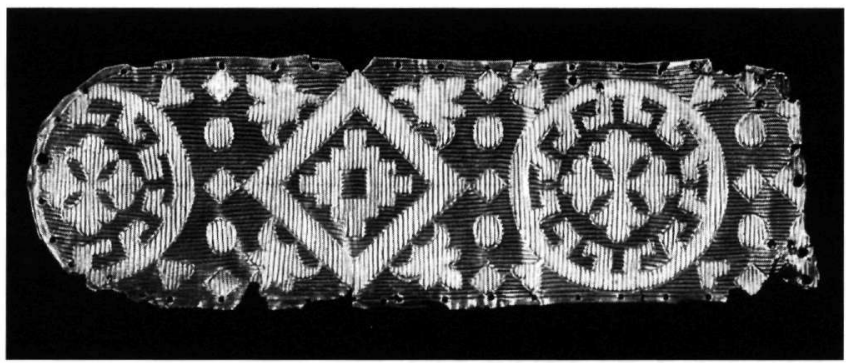

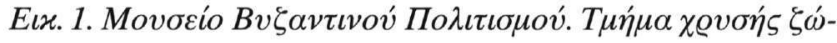

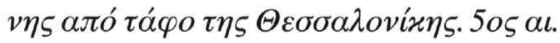

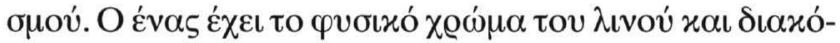

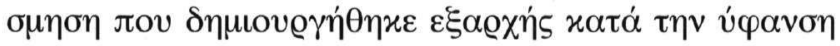

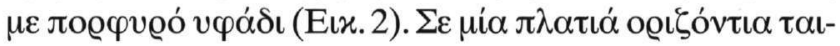

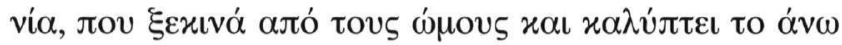

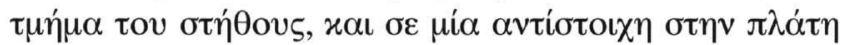

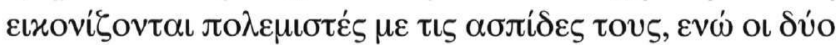

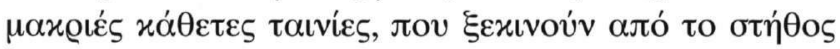

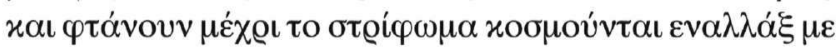

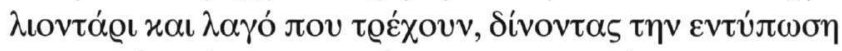

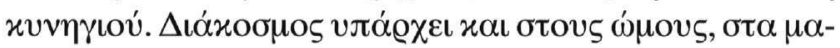

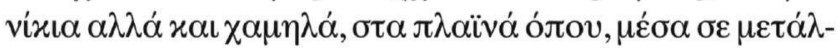

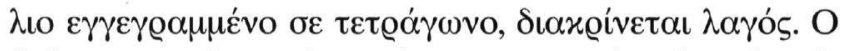

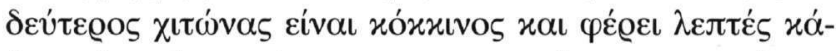

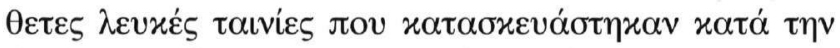

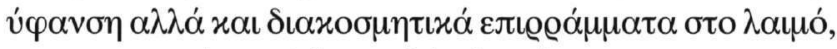

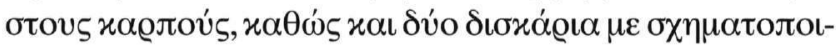

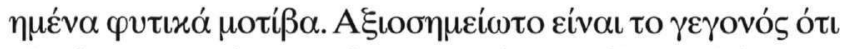

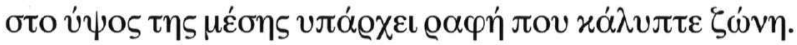

$\gamma$. I $\mu \alpha \tau_{\tau}$

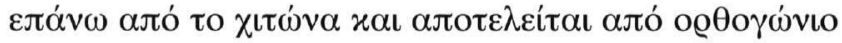

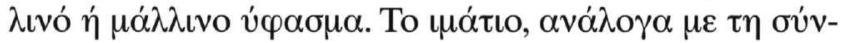

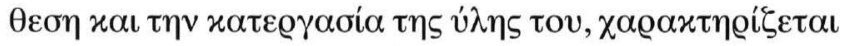

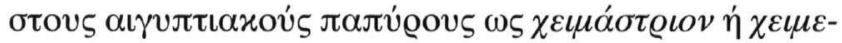

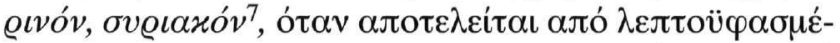

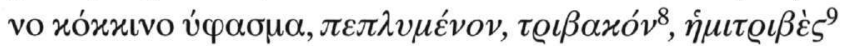

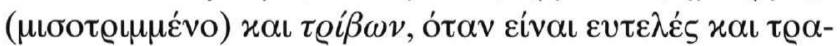

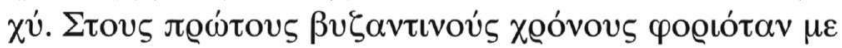

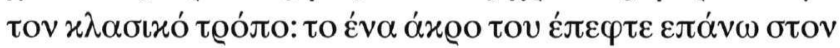

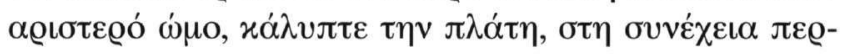

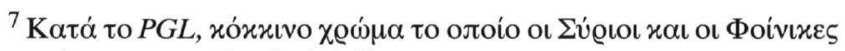

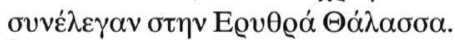

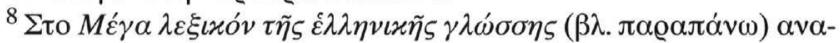

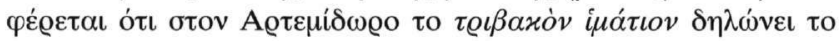

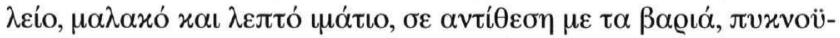

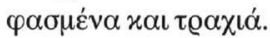

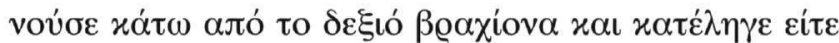

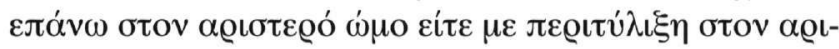

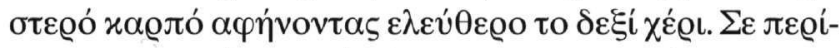

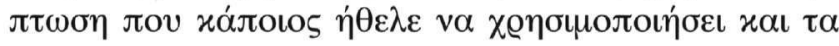

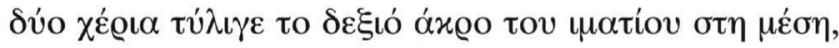

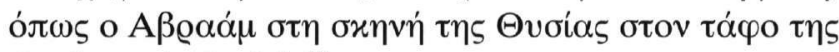
$\Theta \varepsilon о \lambda о \gamma \iota x \dot{\varsigma} \varsigma \Sigma \chi 0 \lambda \varsigma^{10}$.

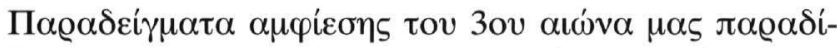

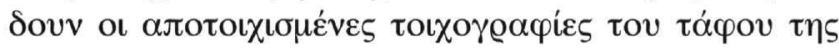

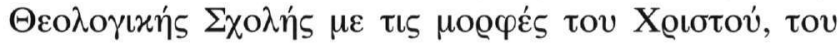

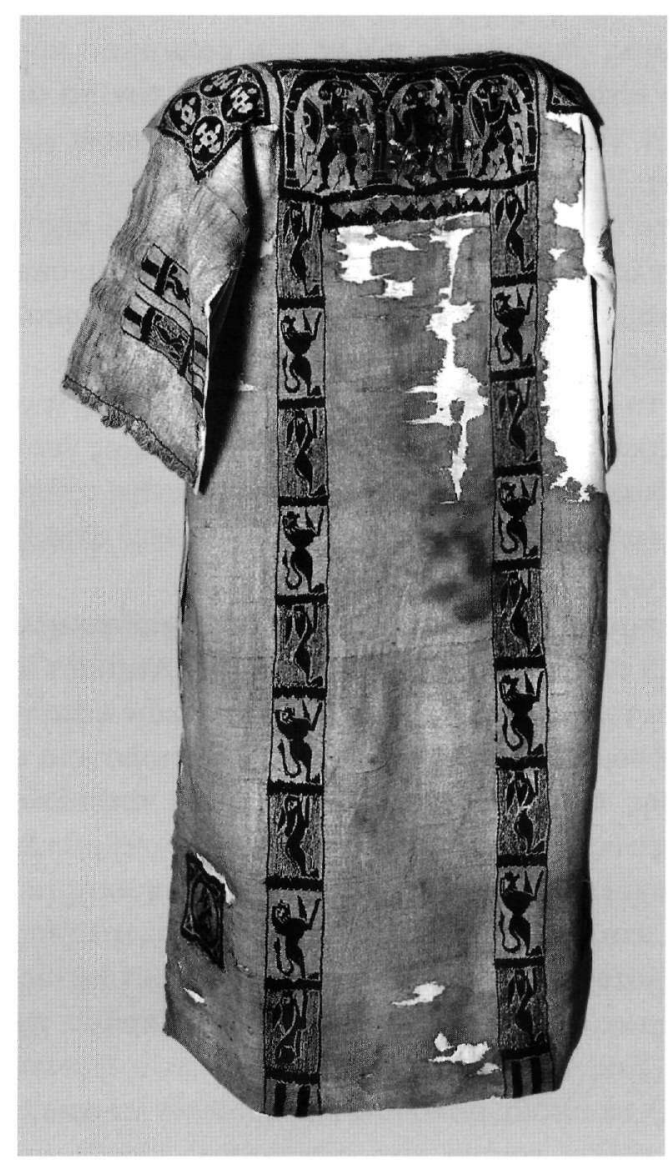

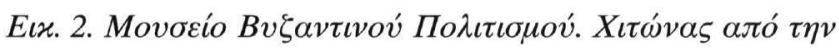

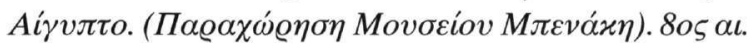

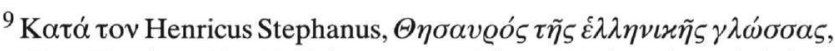

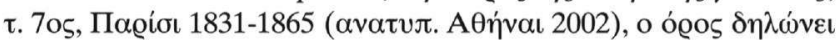

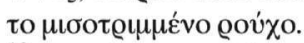

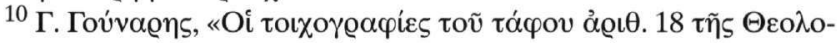

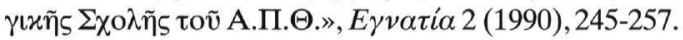




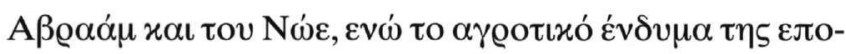

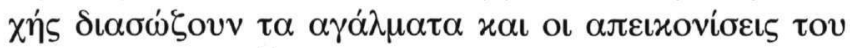

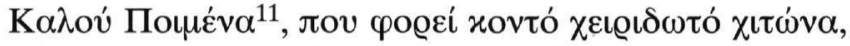

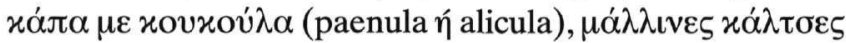

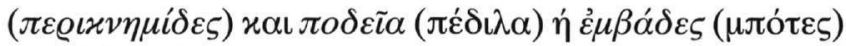

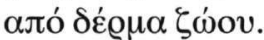

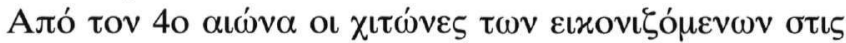

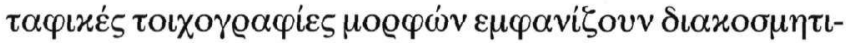

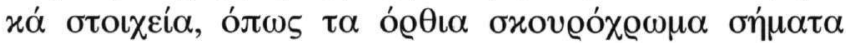

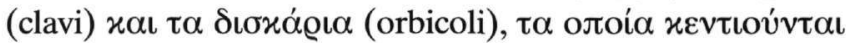

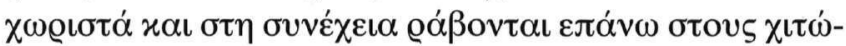

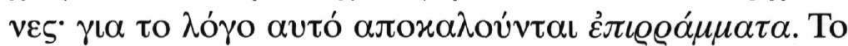

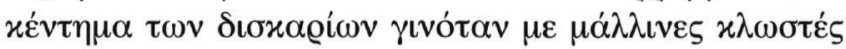

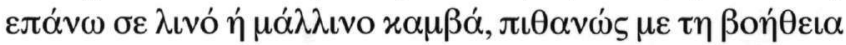

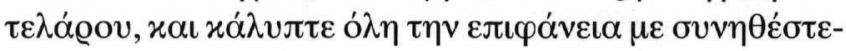

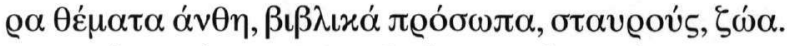

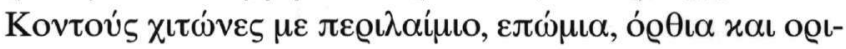

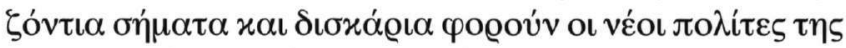

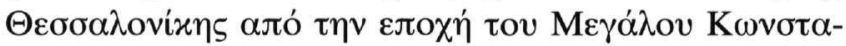

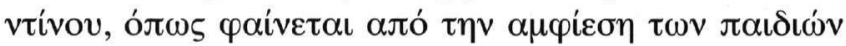

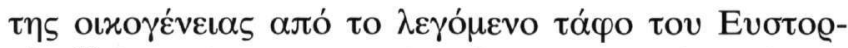

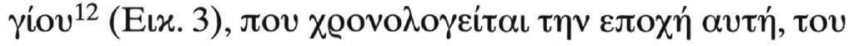

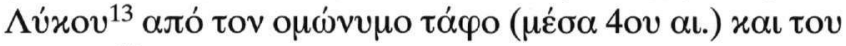

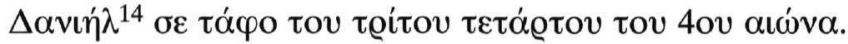

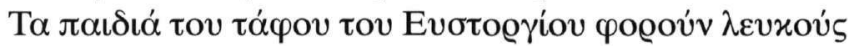

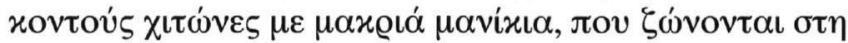

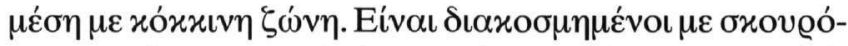

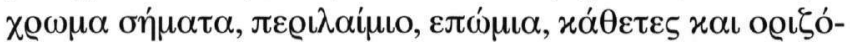

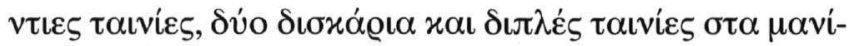

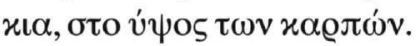

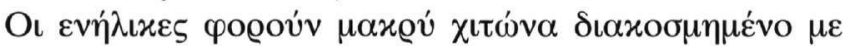

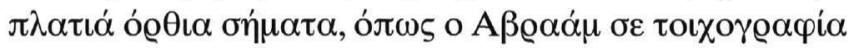

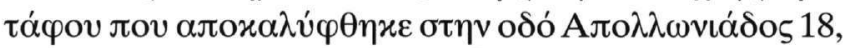

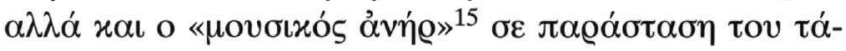

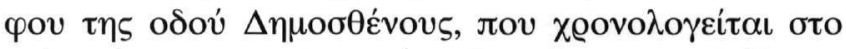

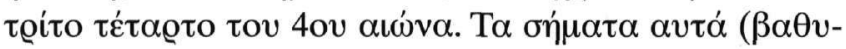

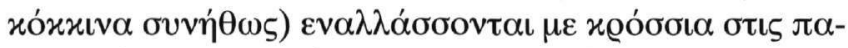

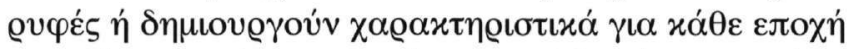

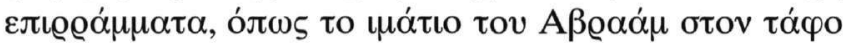

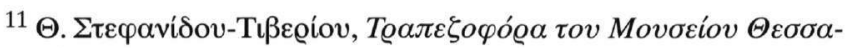

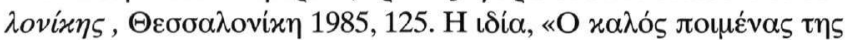

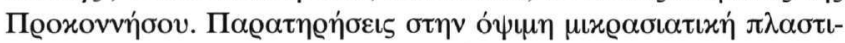

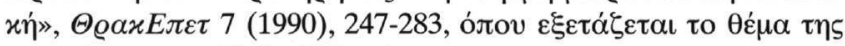

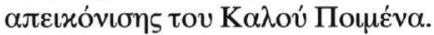

${ }^{12} \mathrm{~S}$. Pelekanidis, «Die Malerei der konstantinischen Zeit», CIAC VII

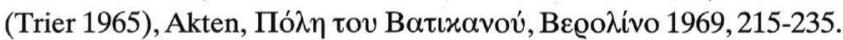

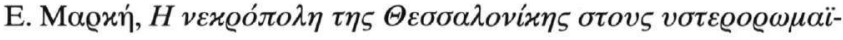

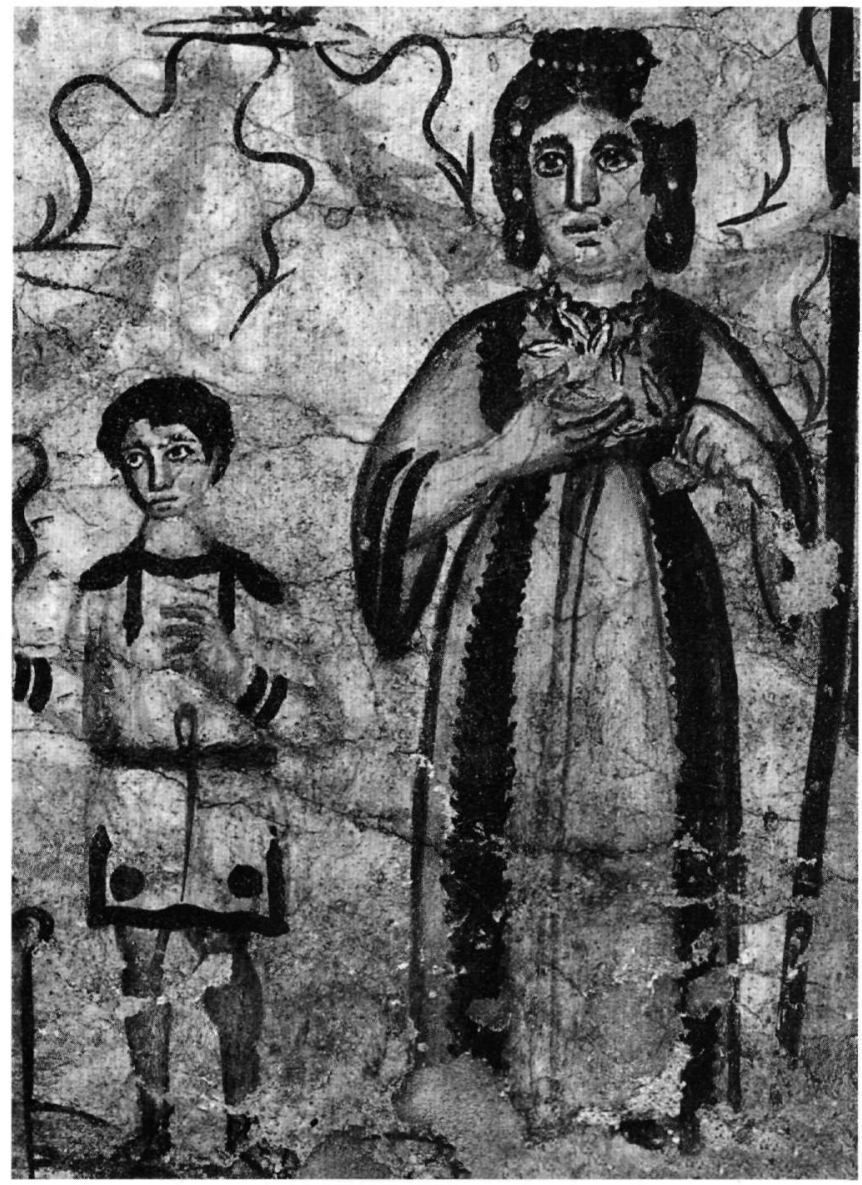

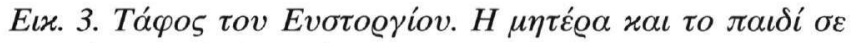

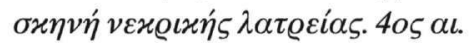

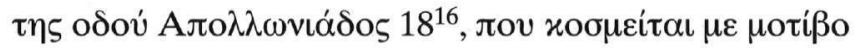

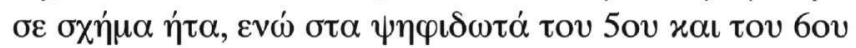

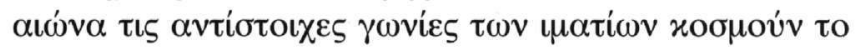

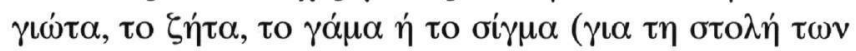
$\alpha \xi \iota \omega \mu \alpha \tau o u ́ \chi \omega \nu \beta \lambda$. $\pi \alpha \varrho \alpha x \alpha ́ \tau \omega)$.

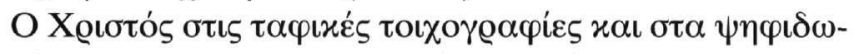

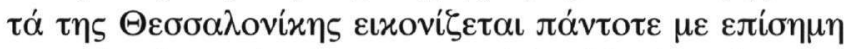

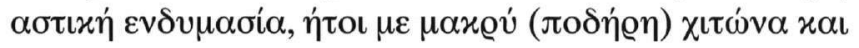

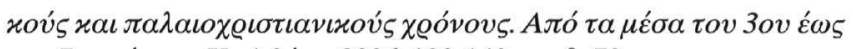

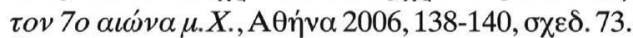

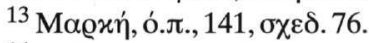

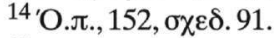

15 'O.л., $152, \sigma \chi \varepsilon \delta .92, \pi i v .11 \gamma-\delta$.

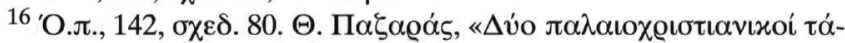

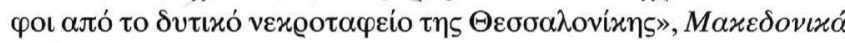
21 (1981), 373-389. 


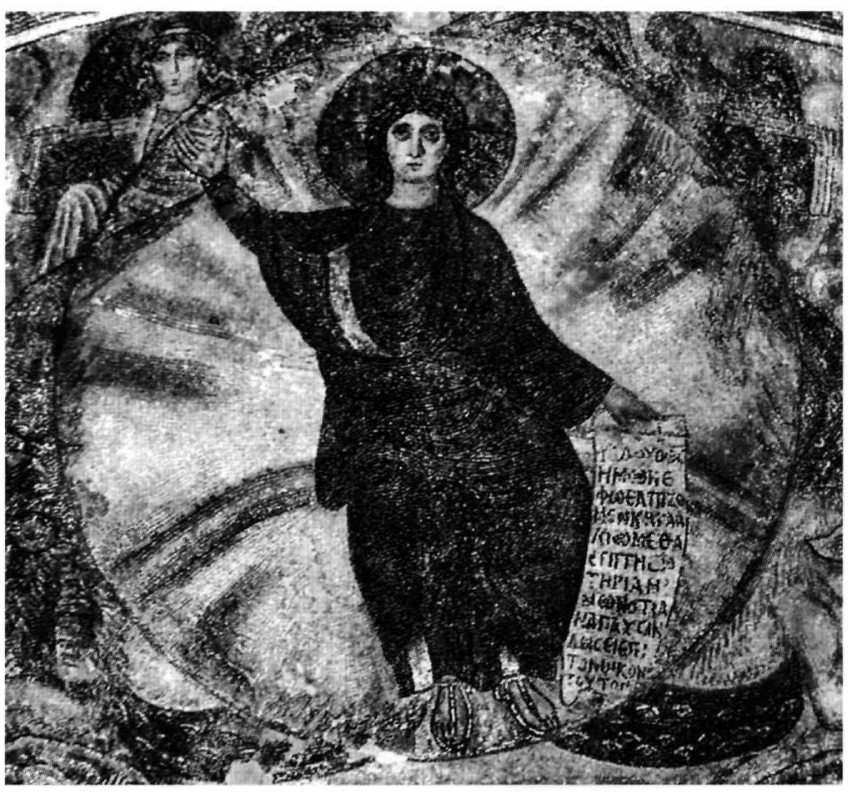

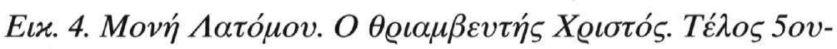
$\alpha \varrho \chi \varepsilon ́ \varsigma 6 o v \alpha \iota$.

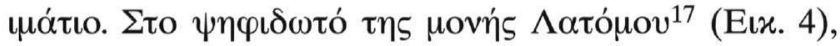

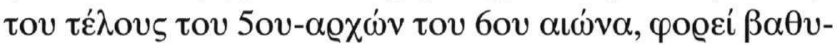

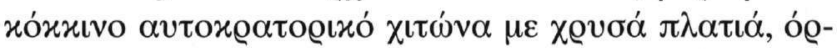

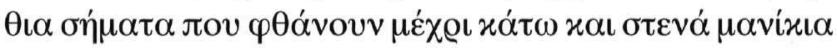

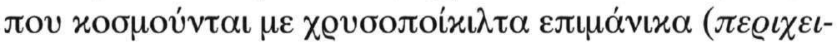

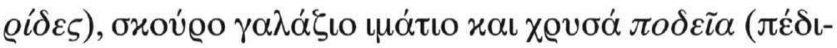

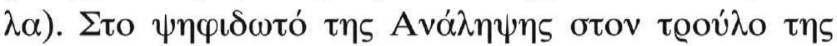

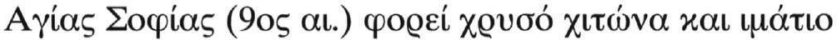

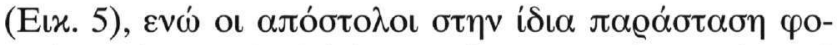

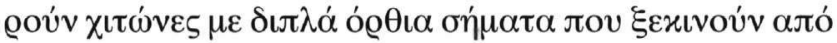

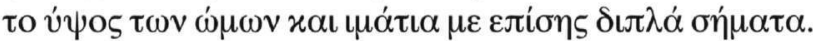

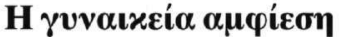

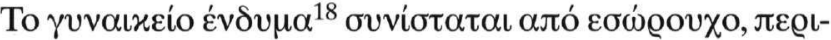

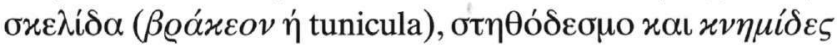

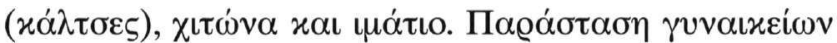
$\varepsilon \sigma \omega \varrho o u ́ \chi \omega \nu ~ \sigma \omega ́ \zeta \varepsilon \tau \alpha \iota ~ \sigma \tau \alpha ~ \psi \eta \varphi \iota \delta \omega \tau \alpha \dot{~} \tau \eta \varsigma$ Piazza Armeri-

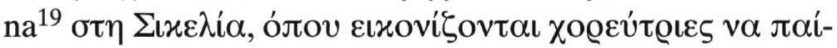

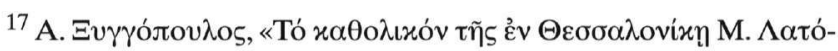

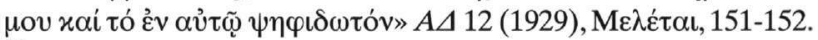

${ }^{18} \mathrm{P}$. Kalamara, Le système vestimentaire à Byzance du IVe jusqu'à la fin

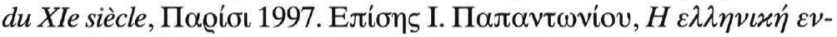

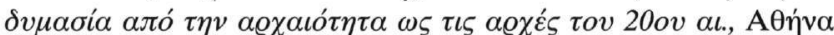
2000. M. Houston, Ancient Greek, Roman and Byzantine Costume and
}

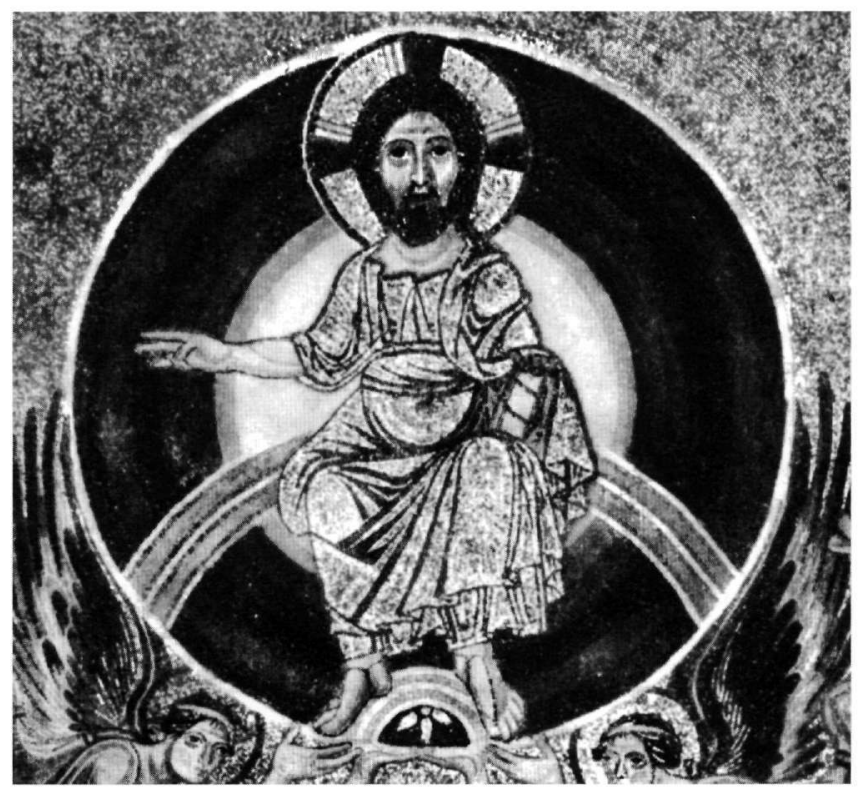

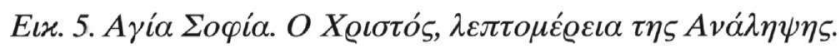
$90 \varsigma \alpha \iota$.

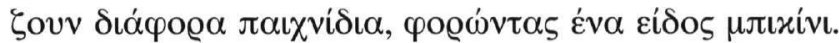

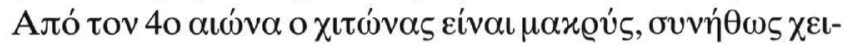

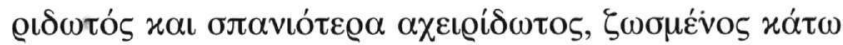

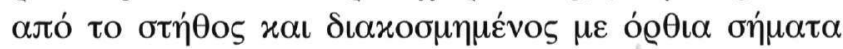

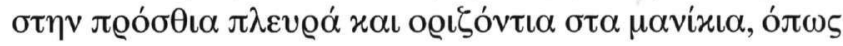

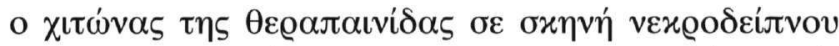

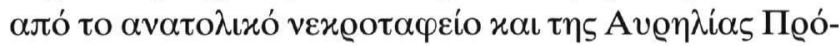

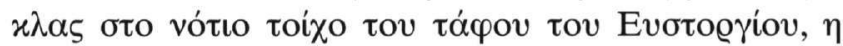
олоі́

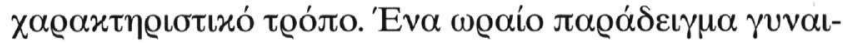

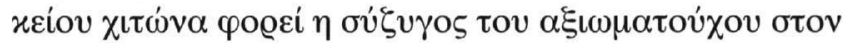

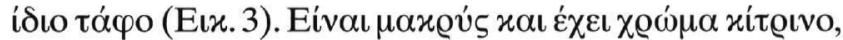

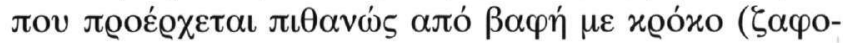

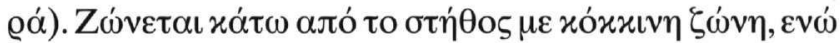

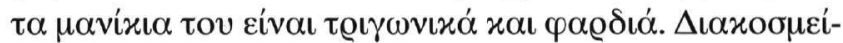

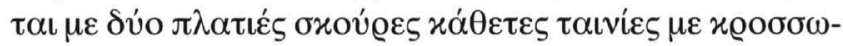

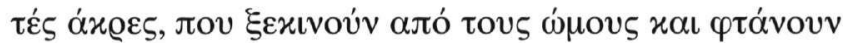

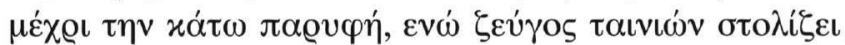

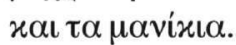

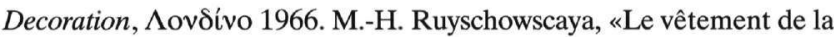
periode copte», Tissus et vetement. 5000 ans de savoir foure, Guity-enVexin 1986.

${ }^{19} \Gamma \alpha \tau \eta v$ Piazza Armerina $\beta \lambda$. A. Garandini - M. Ricci - M. de Vos, Filosophiana. The Villa of Piazza Armerina. The Image of Roman Aristocrat at the Time of Constantin, Palermo 1982. 


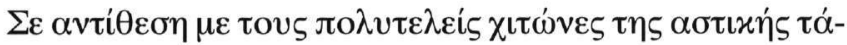

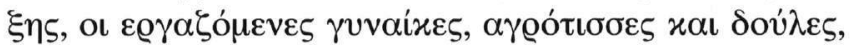

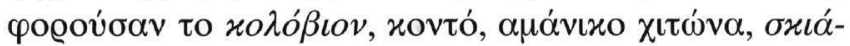

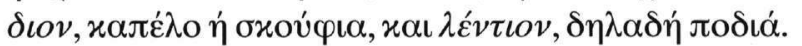

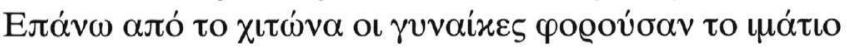

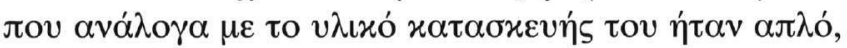

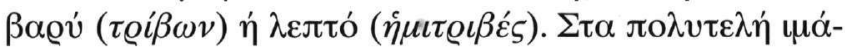

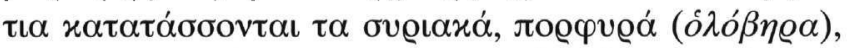

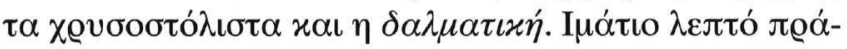

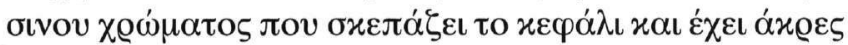

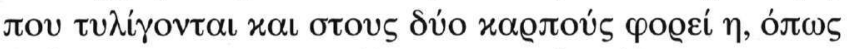

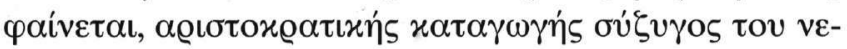

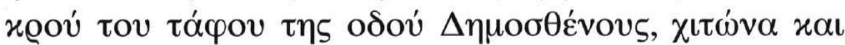

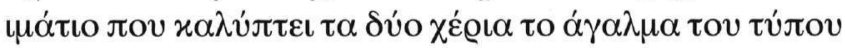

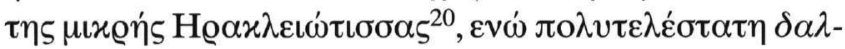

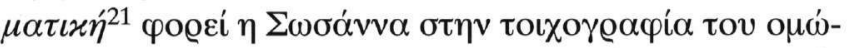

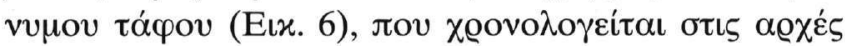

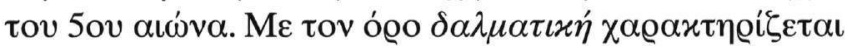

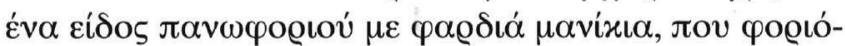

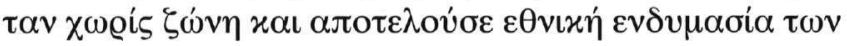

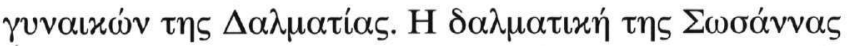

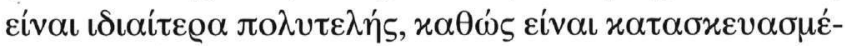

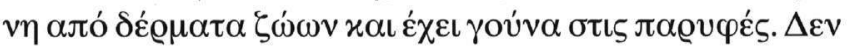

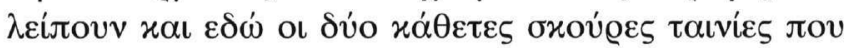

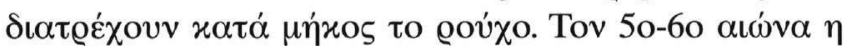

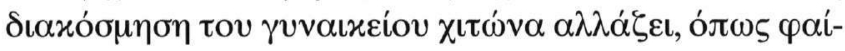

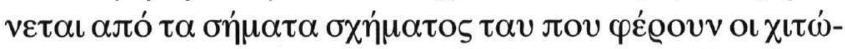

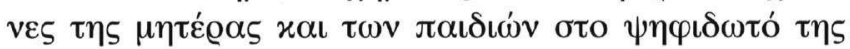

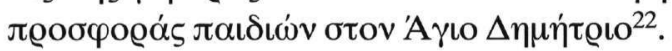

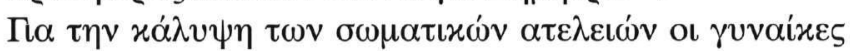

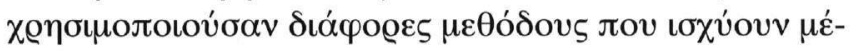

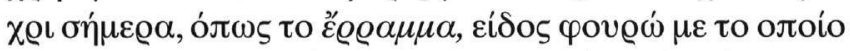

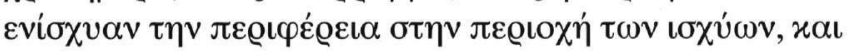

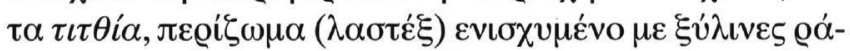

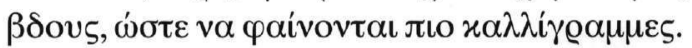

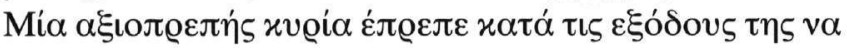

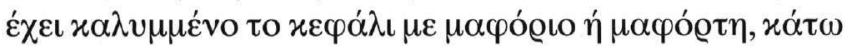

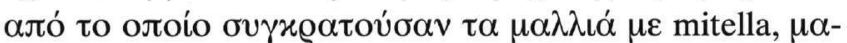

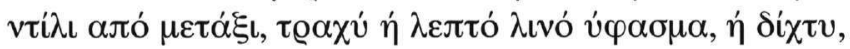

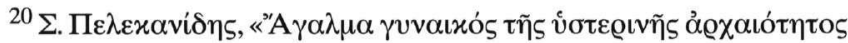

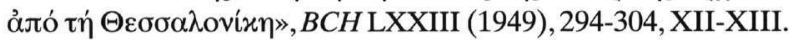

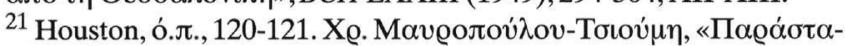

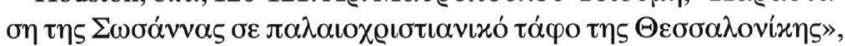

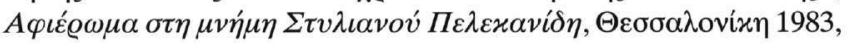
247-258.

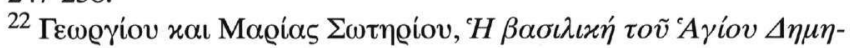

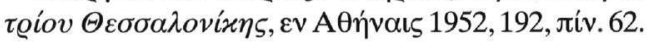

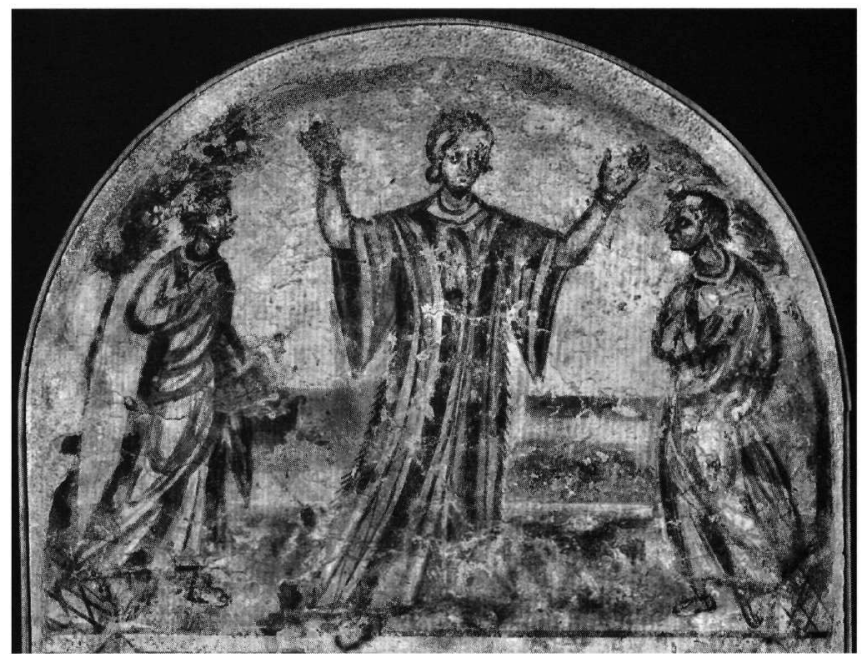

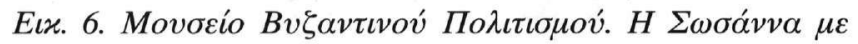

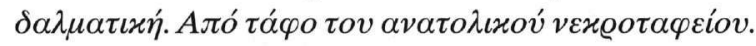

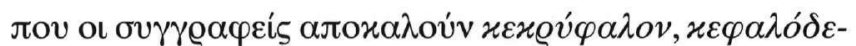

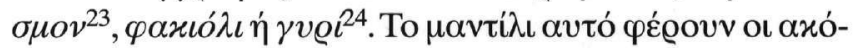

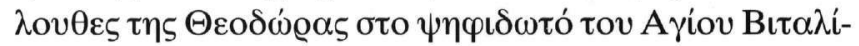

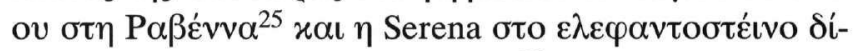

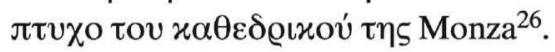

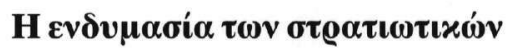

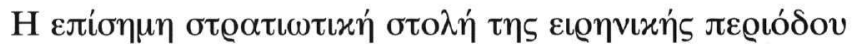

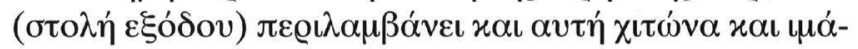

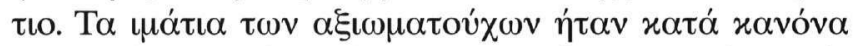

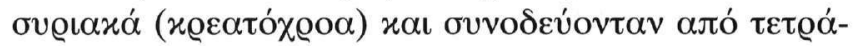

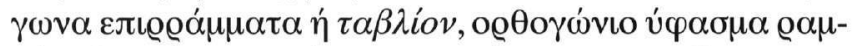

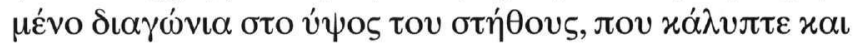

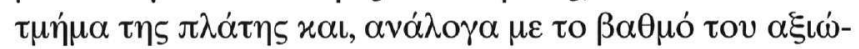

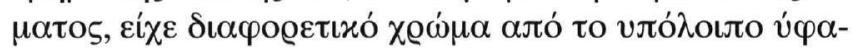

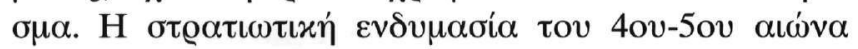

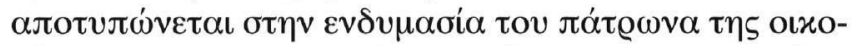

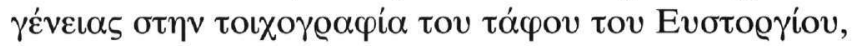

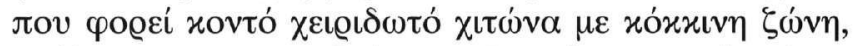

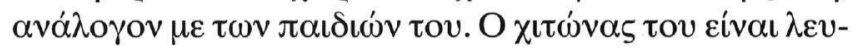

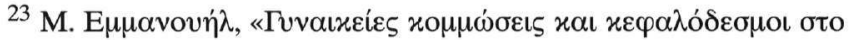

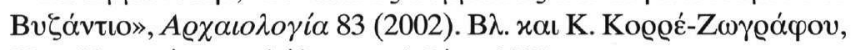

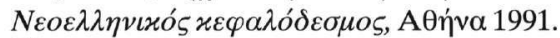

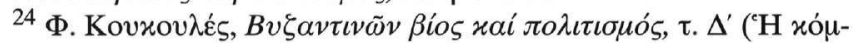

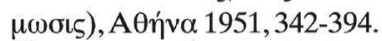

${ }^{25}$ Ravenna Felix, Ravenna 1977, 70-73.

26 W. F. Volbach, Elfenbeinarbeiten der Spätantike und des frühen

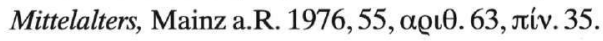




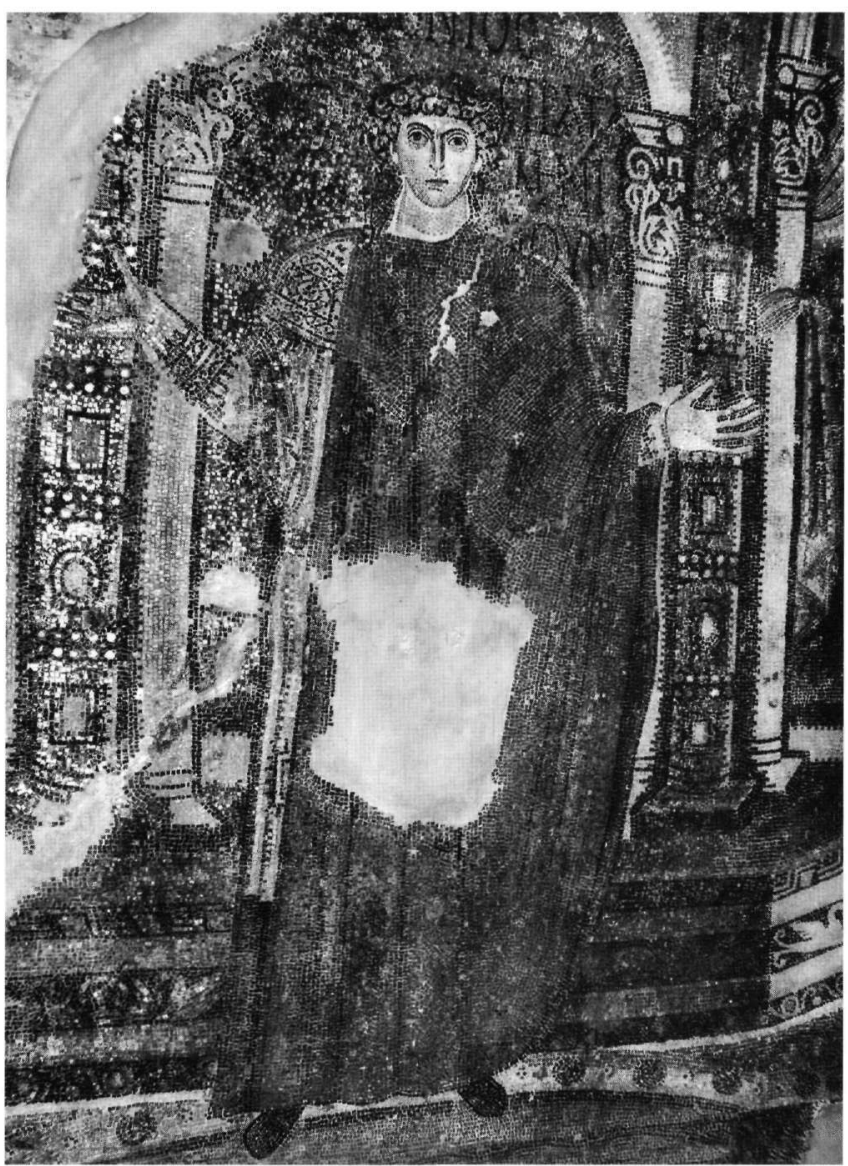

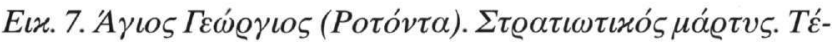
$\lambda o 540 v \alpha$.

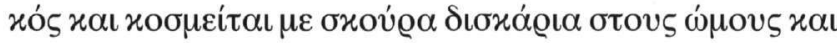

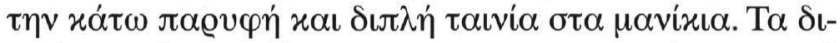

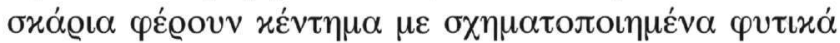

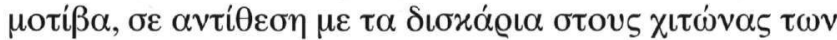

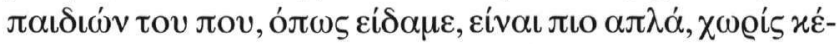

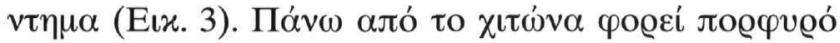

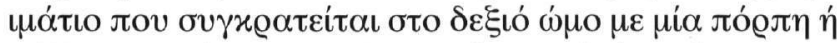

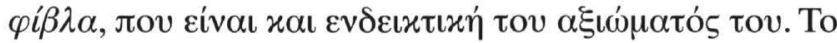

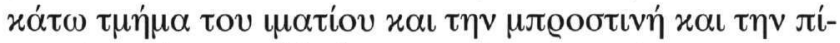

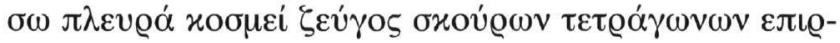

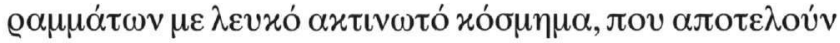

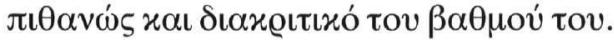

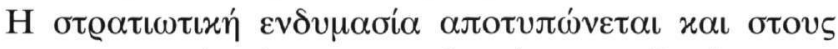

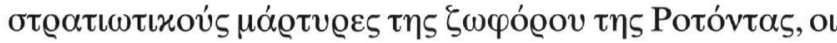

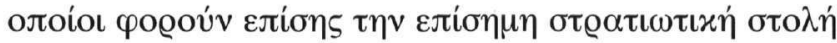

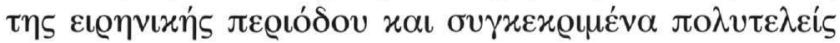

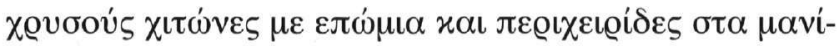

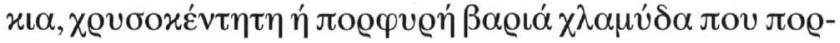

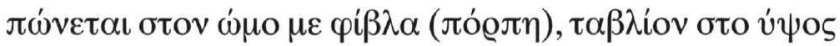

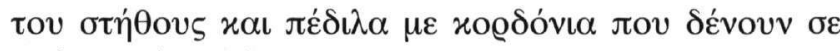
$\sigma \chi \eta \mu \alpha \chi\llcorner($ Еıx.7).

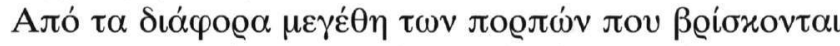

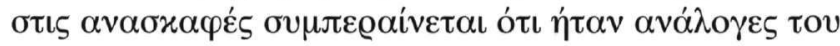

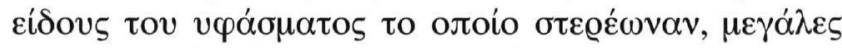

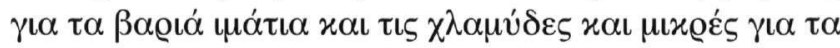

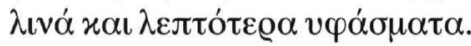

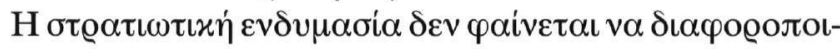

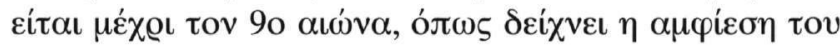

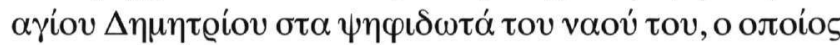

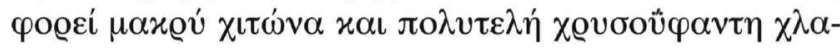

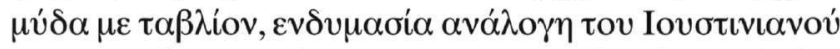

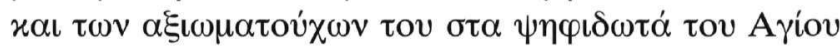

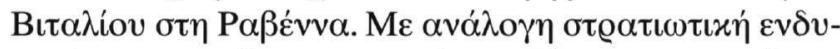

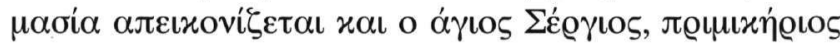

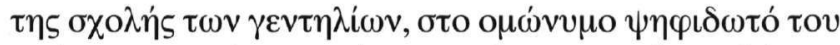

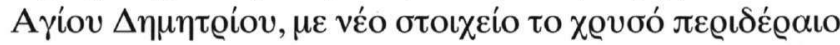

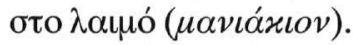

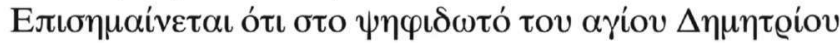

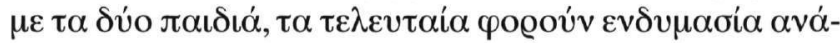

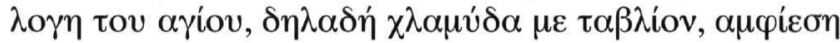

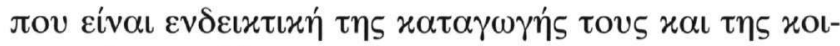

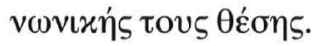

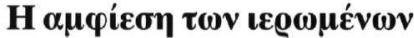

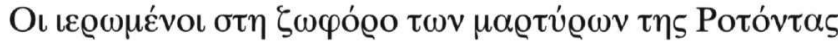

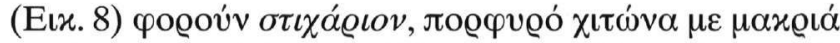

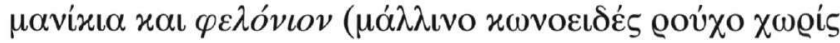

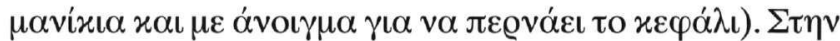

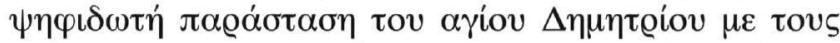

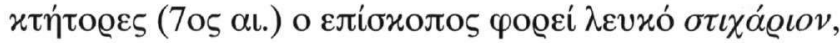

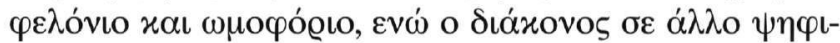

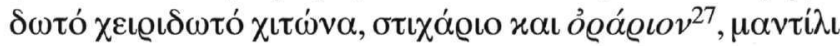

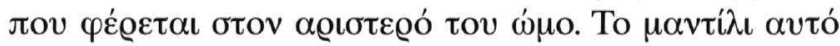

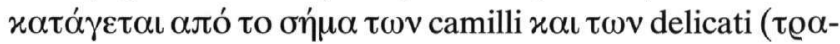

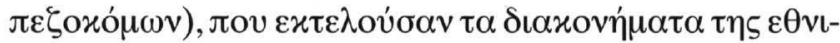

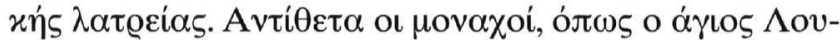

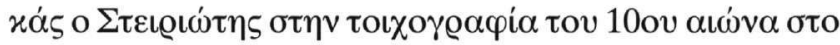

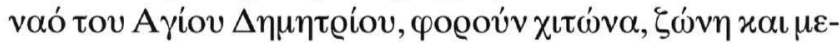

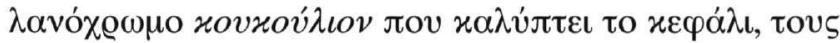

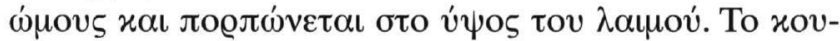

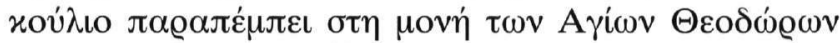

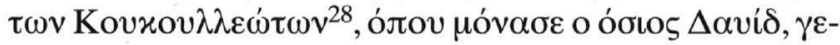

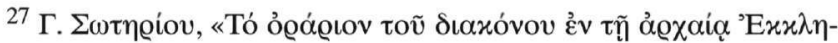
бí(»), $E E \Theta \Sigma \Pi A \mathrm{~A}^{\prime}$ (1926), 405.

${ }^{28}$ R. Janin, Les églises et les monastères des grands centres byzantins, $\Pi \alpha$ -

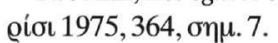




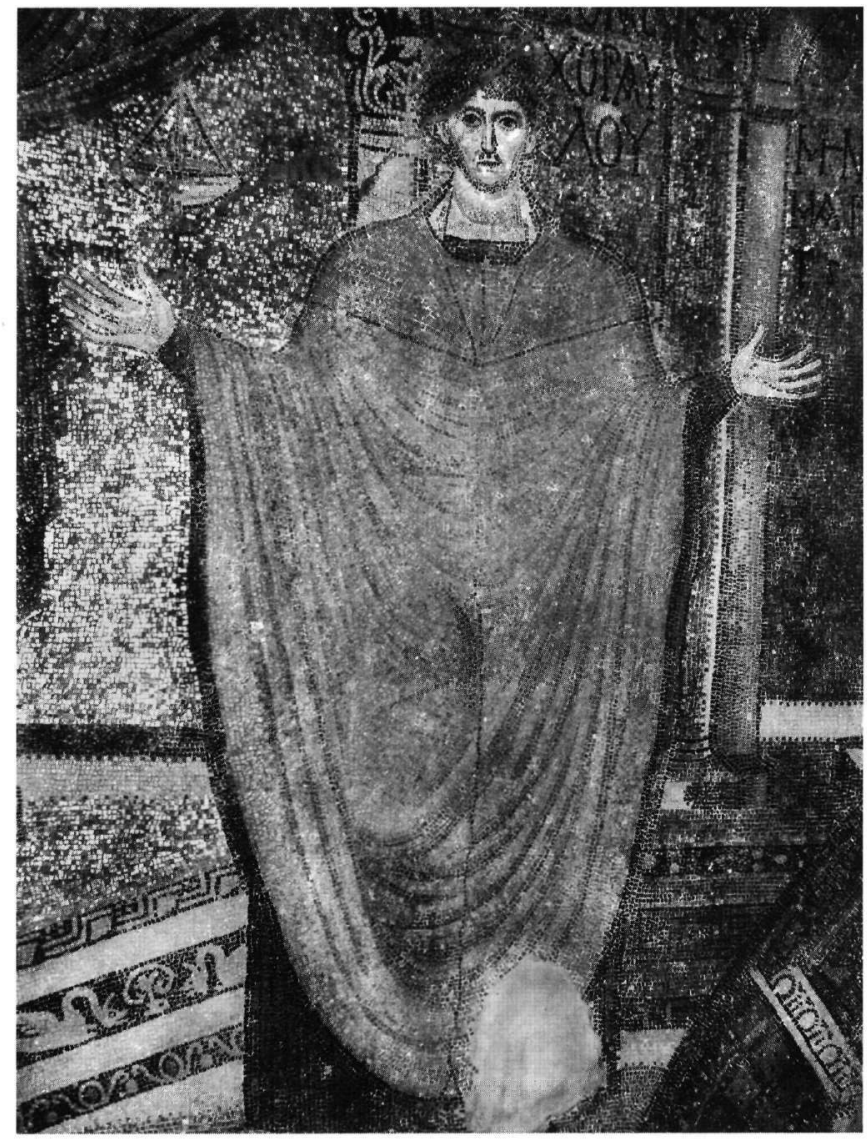

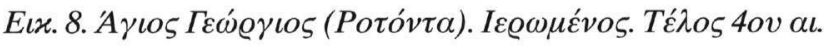

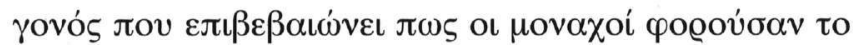

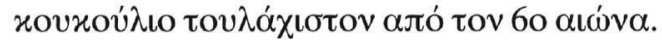

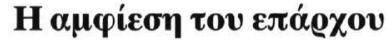

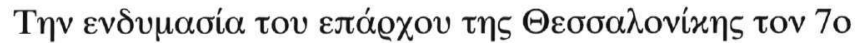

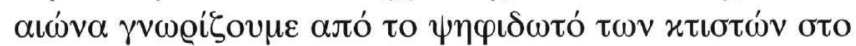

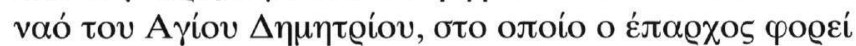

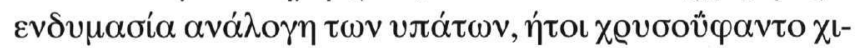

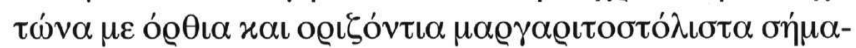

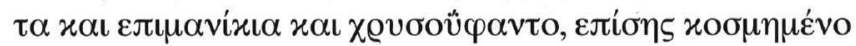

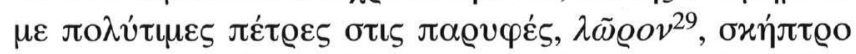

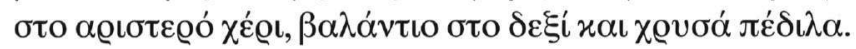

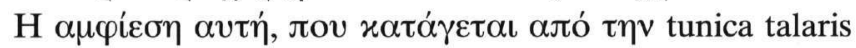

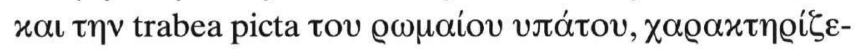

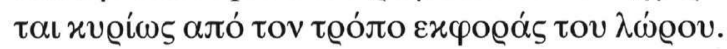

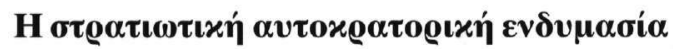

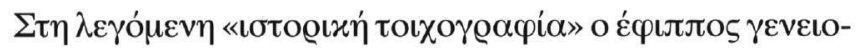

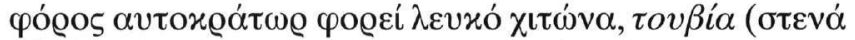
$\pi \alpha v \tau \varepsilon \lambda o ́ v ı \alpha)$ xal $\beta \alpha \theta u ́ \chi \varrho \omega \mu \eta ~ \chi \lambda \alpha \mu v ́ \delta \alpha, \pi o v ~ \alpha \varphi \eta ́ v \varepsilon \iota ~ v \alpha$

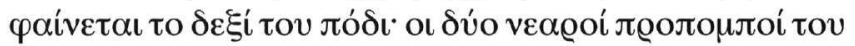

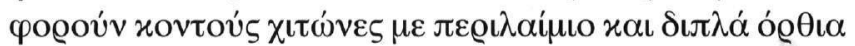

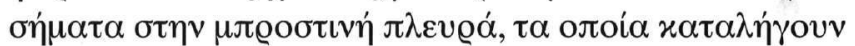

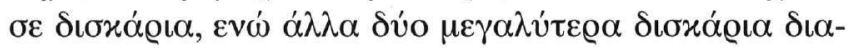

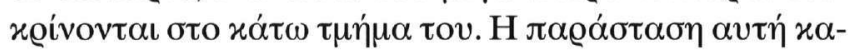

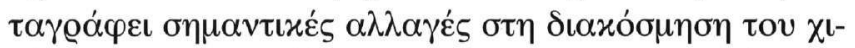

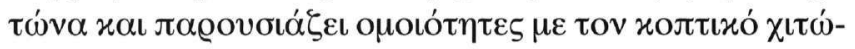

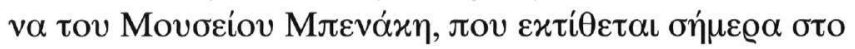

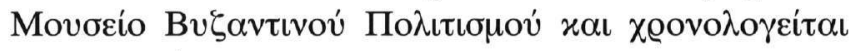

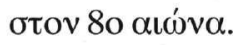

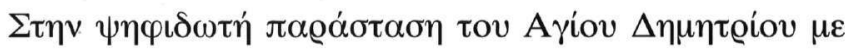

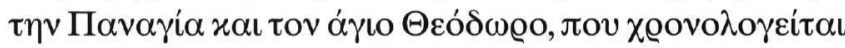

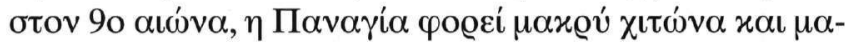

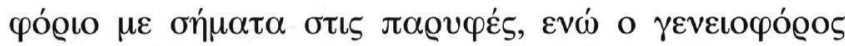

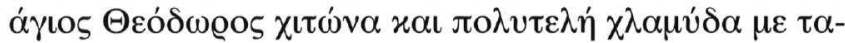

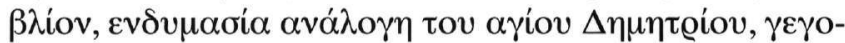

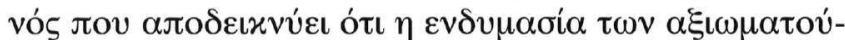

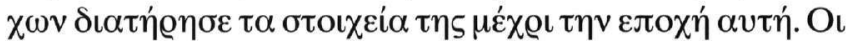

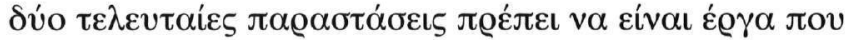

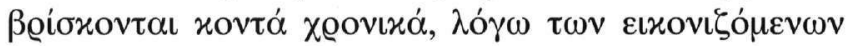

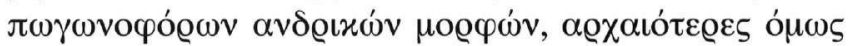

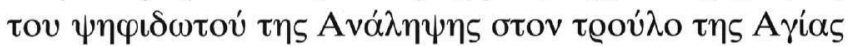

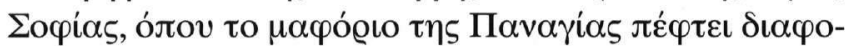

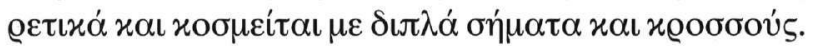

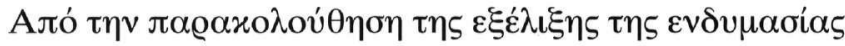

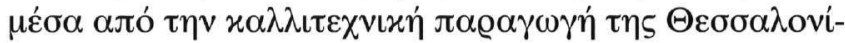

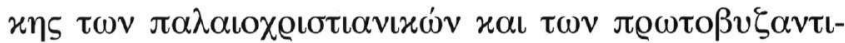

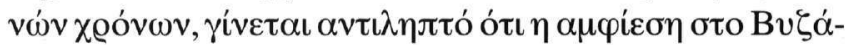

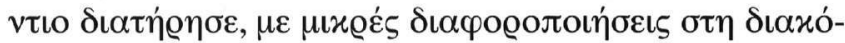

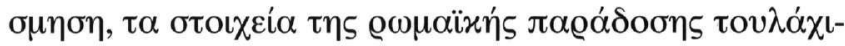

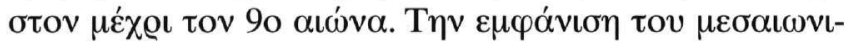

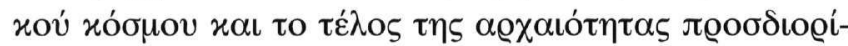

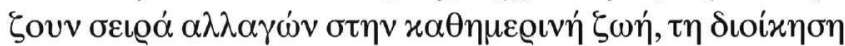

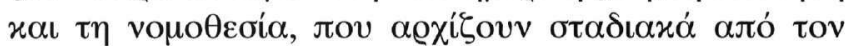

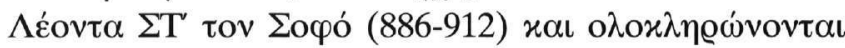

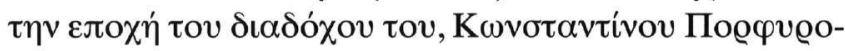

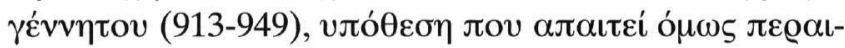

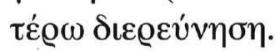

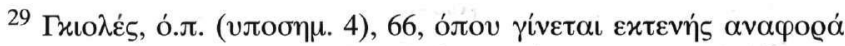

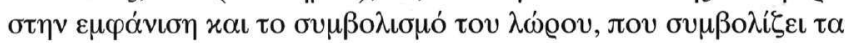

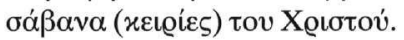




\section{E. Marki}

\section{DRESS IN THE EARLY CHRISTIAN ART OF THESSALONIKI}

\begin{abstract}
$T_{\text {he form and evolution of dress in Thessaloniki in the }}$ Early Christian period are traced through the preserved archaeological remains (pieces of textile, jewellery) and the corresponding art. The archaeological finds come mainly from tombs and consist of a few wretched fragments embroidered with fine cords ('kordonaki' technique), that were sewn to fabrics, fragments of dresses made of gold thread, and gold belts (Fig. 1).

The surviving works of art reveal that the dress of the figures depicted in the art of Thessaloniki was no different from that of the rest of the Roman world, and consists of trousers, chiton (of linen or wool) and himation. Chitons are decorated with clavi and orbicoli (Figs 2 and 3), while vertical clavi and attached pieces of cloth in dark colours are found on himatia. Rural dress is preserved in statues of the Good Shepherd, who wears a short, girt chiton and a hooded cloak, woollen socks and boots.
\end{abstract}

Women's dress includes a short chiton, with or without sleeves, girt beneath the breast and decorated with vertical clavi, himation, and dalmatic made of hide trimmed with fur, like the one worn by Susanna in the wall-painting found in the Tomb of Susanna (Fig. 6), and a headdress for old women.

The praying martyrs in the Rotunda, who wear luxurious chitons with pieces of cloth sewn to the shoulders, and a heavy, gold-embroidered cloak fastened on the shoulder and with a tablion sewn on the breast illustrate military dress (Fig. 7). The priests in the same frieze wear a phelonion, sticharion and omophorion (Fig. 8), while the official dress of the eparchos is preserved in the founder's mosaic in Ayios Dimitrios: it comprises a gold chiton with vertical clavi, epimanikia, a gold-woven loros, gold sandals, sceptre and purse. In the mosaic depicting the saint of this church, the deacon wears a sticharion and orarion, a kind of stole worn over the left shoulder.

In the historical wall-painting in Ayios Dimitrios, a representation in which important changes in military uniform are recorded, the mounted, bearded emperor wears a short chiton, socks and cuirass, and his two escorts have similar chitons with a collar and double vertical clavi on the front that end in orbicoli. Finally, in the mosaic of the Virgin and St Theodore in Ayios Dimitrios, the Virgin wears a long chiton and maphorion and the bearded Theodore has a chiton and a yellowish cloak with a tablion. The last two representations are dated to the same period, the 8th-9th century, on the basis of the bearded figures depicted.

Study of the above evidence, archaeological finds and artworks of Thessaloniki reveals that dress in this city retained the features of the Roman tradition at least down to the 8th9 th century. 